\title{
Highly Directional Fluorescent Emission at Visible Wavelengths with a Silicon Metasurface
}

\author{
Wuzhou Song ${ }^{1}$, Shi-Qiang $\mathbf{L i}^{2}$, and Kenneth B. Crozier*1,2 \\ ${ }^{1}$ School of Physics, University of Melbourne, VIC 3010, Australia \\ ${ }^{2}$ Department of Electrical and Electronic Engineering, University of Melbourne, VIC 3010, Australia \\ *-Email: kenneth.crozier@unimelb.edu.au
}

\begin{abstract}
We experimentally demonstrate, for the first time to our knowledge, highly-directional fluorescence emission at visible wavelengths from a silicon metasurface covered with a dye-doped thin film. We provide a physical interpretation based on multipole expansion.

OCIS codes: (350.4238) Nanophotonics and photonic crystals; (160.3918) Metamaterials
\end{abstract}

An improved ability to shape the radiation patterns of dipole emitters would be beneficial for many applications. This has led to much interest in nano-optical approaches such as Yagi-Uda nanoantennas [1], and plasmonic bullseyes [2] and plasmonic crystals [3]. Impressive results have been obtained, but challenges remain. Previous devices: 1). often impose significant restrictions on the emitter position and orientation 2). often operate over narrow bandwidths, sometimes in the infrared, and 3). can exhibit low efficiency due to ohmic losses in metallic elements. The dielectric metasurface has recently emerged as an approach by which optical devices such as lenses can be achieved in a highly flexible manner [4]. In this work, for the first time to the best of our knowledge, we experimentally demonstrate highly directional fluorescent emission using an all-dielectric metasurface at visible wavelengths. It comprises silicon ( $\mathrm{Si}$ ) nanorods that act as a passive phased array.

Fabrication begins with a silicon-on-insulator (SOI) wafer, whose top $\mathrm{Si}$ is thinned to $160 \mathrm{~nm}$. Nanorods are defined by electron beam lithography and dry etching in square arrays (period: $300 \mathrm{~nm}$ ). The bulk (handle) Si of the SOI wafer is removed by etching, and the nanorods are transferred to a glass wafer using UV glue. The nanorods are then spin coated with polymethylmethacrylate (PMMA, thickness $\approx 180 \mathrm{~nm}$ ) doped with the fluorescent dye Nile-red. Fig. 1 shows the reflectance spectrum of the metasurface before coating. Measured and simulated reflection spectra are in good agreement, with two peaks corresponding to electric and magnetic dipole nanorod resonances. In the area labeled "reference" in Fig. 1, the top Si has been etched down to the oxide. We next perform fluorescence microscopy with green excitation (filtered LED source) and a microscope objective (10×, NA: 0.25$)$. The fluorescent microscopy image (Fig. 2 left inset) shows significantly stronger emission (up to $\sim 7$ times) from the metasurface than the reference. Measured fluorescence spectra (Fig. 2) indicate that the largest enhancement is at $\lambda=590 \mathrm{~nm}$, and that there is some spectral narrowing. Fluorescence lifetime measurements (Fig. 2 right inset) exclude the Purcell effect as the origin of the observed enhancement. We next measure far-field emission patterns using fluorescence microscopy as before, but with focused green laser excitation $(\lambda=532 \mathrm{~nm}$ ), an oil immersion objective lens (NA: 1.40), and by displacing the camera from the focal plane. The device is covered with oil. The results are shown as Fig. 3, with the upper and lower parts measured without and with emission filtering by a laser line filter (center wavelength: 590nm, FWHM: $10 \mathrm{~nm}$ ), respectively. It can be seen that the fluorescent emission is much more directional with the metasurface, and with greater peak intensity. For comparison of the peak intensities from the metasurface and reference, Fig. 3c $\& \mathrm{f}$ should be referred to, as the color-scale of each pattern of Fig. 3a,b,d,e is independently normalized. The results with the laser line filter (Fig. 3d-f), show an intense ring shape projection pattern with a peak intensity $\sim 8$ times larger than the reference. For physical interpretation, we perform finite difference time domain (FDTD) simulations of the far-field pattern produced by an electric dipole source located in a nanorod array (Fig. 4). It can be seen that directional emission is achieved for all source dipole orientations. FDTD simulations also predict that substantial displacement of the emitter modifies the emission pattern, but that it remains highly directional (Fig. 5). To further understand the role of the nanorods, we perform multipole expansion [5] for the case of the source dipole being centrally located (Fig. 4a). The results (Fig. 6a) reveal that the electric (ED) and magnetic dipoles (MD) dominate the response. In Fig. 6b, we show the strengths of the ED and MD dipoles for nanorods located at increasing distance from the source dipole, revealing that only three specific dipoles dominate in the entire array. Fig. $6 \mathrm{c}$ and $\mathrm{d}$ plot the intensity and phase distributions of $\mathrm{ED}_{\mathrm{z}}$ for a dipole source centrally located in an $10 \times 10$ array of nanorods. It can be seen that the closest few nanorods dominate the response.

We acknowledge funding from the Laby Foundation, VESKI, and Australian Research Council.

\section{References}

[1] A. G. Curto et al, "Unidirectional emission of a quantum dot coupled to a nanoantenna" Science 329, 930-933(2010)

[2] H. Aouani et al. "Plasmonic Antennas for Directional Sorting of Fluorescence Emission" Nano. Letts., 11, 2400-2406 (2011) 
[3] G. Lozano et al, "Plasmonics for solid-state lighting: enhanced excitation and directional emission of highly efficient light sources" Light: Science \& Applications 2, 66 (2013)

[4] S. Jahani and Zubin Jacob "All-dielectric metamaterials" Nat. Nano., 11, 23-46 (2016)

[5] P. Grahn et al, "Electromagnetic multipole theory for optical nanomaterials", New J. Phys. 14, 093033 (2012)

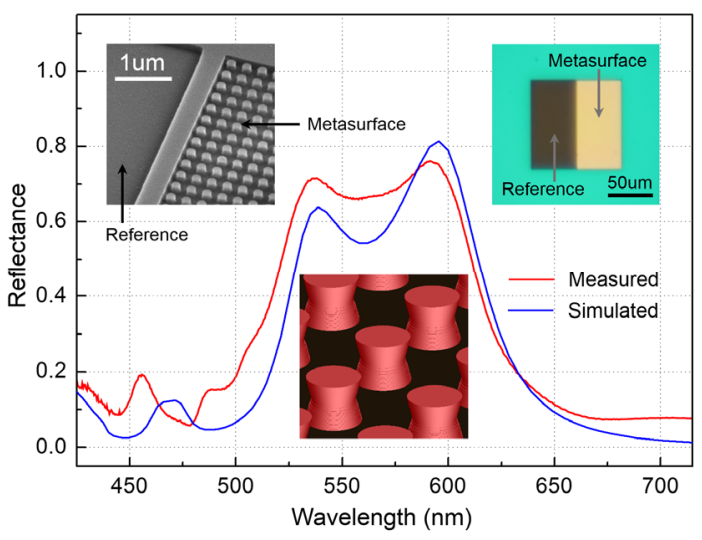

Fig. 1. Reflectance spectra of metasurface. Left and right insets show scanning electron microscope (SEM) and bright field microscopy images of the device. Middle bottom inset shows the nanorod shape that is simulated (upper diameter of nanorod: $148 \mathrm{~nm}$, middle: $120 \mathrm{~nm}$, bottom: $152 \mathrm{~nm}$ )

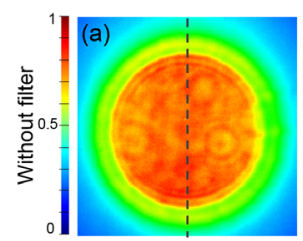

Reference

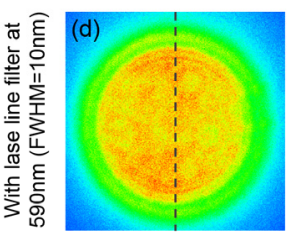

Reference

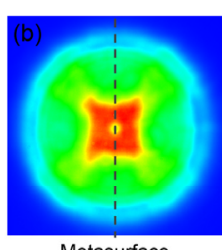

Metasurface

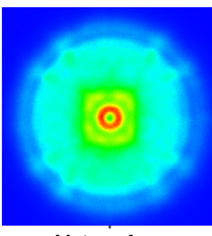

Metasurface
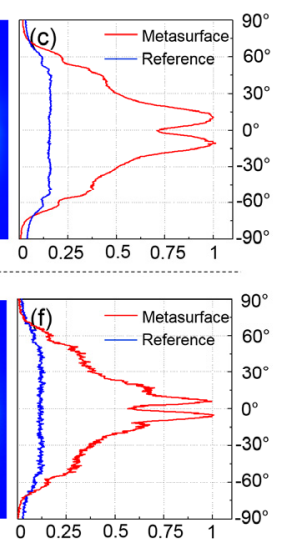

Fig. 3. Far field fluorescence emission patterns and their crosssectional profiles. Upper and lower parts show patterns without \& with adding the laser line filter. (c)) and (f) are the crosssectional profile extracted from data along dotted lines.
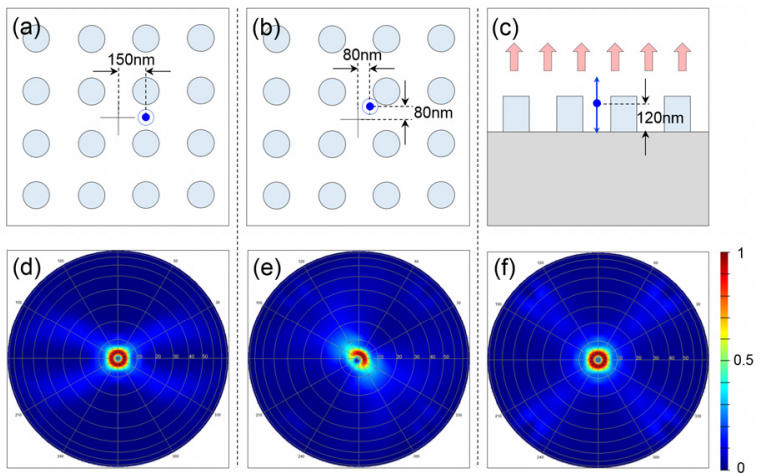

Fig. 5. FDTD simulation results of far field radiation patterns for source electric dipole located in different positions. In (a, d) and (b, e), the dipole is located at a height of $80 \mathrm{~nm}$ above the substrate

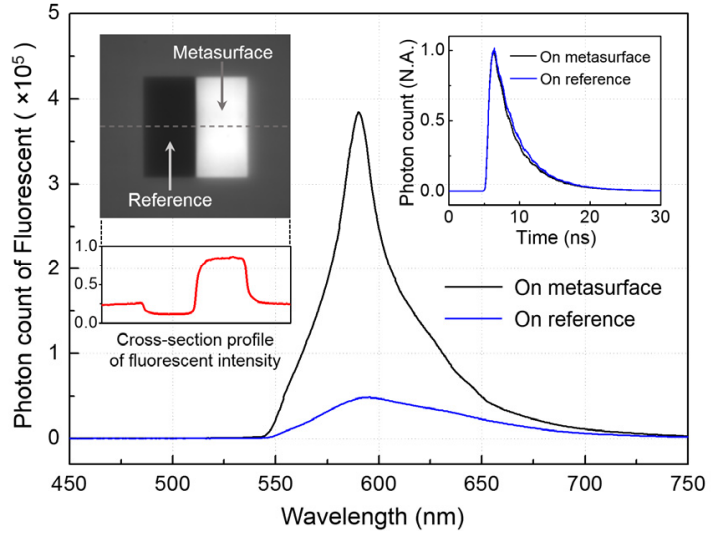

Fig. 2. Fluorescence spectra measured from metasurface and from reference (with NA 0.25 objective lens). Left inset: fluorescence microscopy image. Right inset: fluorescence decay curves measured from metasurface $\&$ from reference..
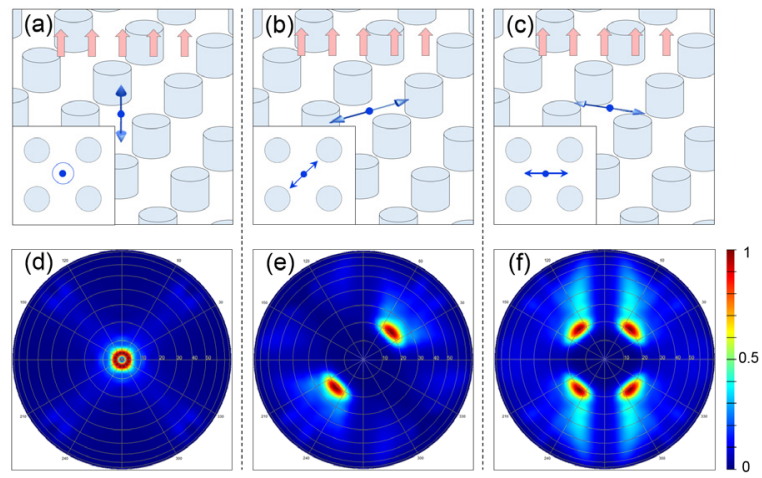

Fig. 4. FDTD simulation results of far field radiation patterns for source electric dipole $(\lambda=590 \mathrm{~nm})$ that is centrally located in nanorod array in different orientations
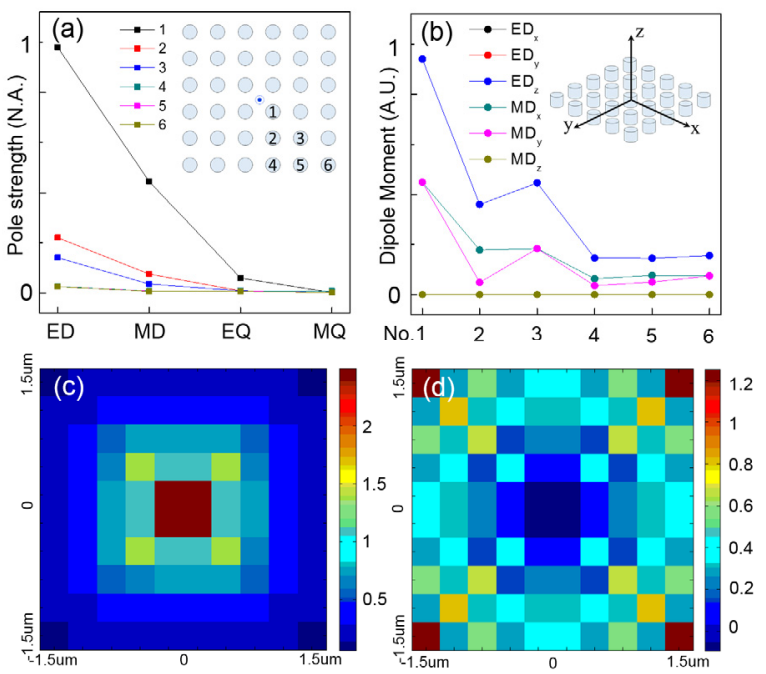

Fig. 6. Multipole expansion results. (a) Strengths of different poles of nanorods at different locations. (b) Comparison of different components of electric \& magnetic dipoles for nanorods in different locations. Distribution of (c) intensity and (d) phase of $E D_{z}$. in array of $10 \times 10$ nanorods. 